\title{
Van der Waals effect in weak adsorption affecting trends in adsorption, reactivity, and the view of substrate nobility
}

\author{
André K. Kelkkanen, ${ }^{1}$ Bengt I. Lundqvist, ${ }^{1,2}$ and Jens K. Nørskov ${ }^{1}$ \\ ${ }^{1}$ Center for Atomic-scale Materials Design, Department of Physics Technical University of Denmark, \\ DK - 2800 Kongens Lyngby, Denmark \\ ${ }^{2}$ Department of Applied Physics, Chalmers University of Technology, SE-41296 Göteborg, Sweden
}

(Received 9 December 2010; published 11 March 2011)

\begin{abstract}
The ubiquitous van der Waals (vdW) force, particularly discernible in weak adsorption, is studied on noble and transition metals. In calculations with the vdW density functional (DF) [M. Dion et al., Phys. Rev. Lett. 92, 246401 (2004)], the atomic structure near the adsorption site is systematically varied, including dense fcc(111) surface, adatom, pyramid, and step defects. In weak adsorption the vdW force (i) is shown necessary to account for, (ii) is sizable, (iii) has a strong spatial variation, relevant for adsorption on surface defects, (iv) changes reaction rules, and (v) changes adsorption trends in agreement with experimental data. Traditional physisorption theory is also given support and interpretation.
\end{abstract}

DOI: 10.1103/PhysRevB.83.113401

PACS number(s): 68.43.-h, 68.47.De, 71.15.Mb, 73.20.-r

When molecules and materials meet, as a molecule hitting a surface in catalysis or a contact between two pieces of materials, the tails of each chunk of electrons guide the further fate of the systems. Often stronger processes take over and the effect gets lost, but not so for an important class of phenomena, here called "weak adsorption," including physisorption and hydrogen bonding.

Well-prepared crystallographically ordered surfaces provide the basis for the accurate comparisons between experiment and theory that stand behind the impressive accomplishments of surface science. The even more general property of a surface, its two-dimensional extent, is profitable for phenomena that gain strength by a surface source, like the ubiquitous van der Waals (vdW) force. In this Brief Report the vdW-force landscapes are calculated for some representative atomic configurations, showing that the extent of surfaces matters, and that they upset some adsorption experiences and reaction rules.

Weak adsorption, with adsorption-energy values less than about $1 \mathrm{eV}$, is abundant, often significant, and encompasses several kinds of forces. Some of them derive from electrostatics and quantum statistics and can be accounted for at the level of the general-gradient approximation (GGA) of density-functional theory (DFT), others derive from quantummechanical correlations, like vdW forces, and surface properties, like lateral forces.

Many catalytic reactions are structure sensitive, rates and binding energies depending on the detailed geometric structure of the surface atoms of the catalysts. ${ }^{1,2}$ At defects, like steps, kinks, edges, and adparticles, atoms and molecules are generally more reactive than on flat surfaces, a fact attributed to an under-coordination of the reacting particle. ${ }^{1-4}$ This can be tied to the altered electron structure at such sites, since the local $d$ band narrows and shifts upward in energy close to the Fermi level, giving stronger bonds for many atoms on such sites than on flat surfaces, as described in the $d$-band model, ${ }^{5,6}$ calculated in GGA, ${ }^{2,7-9}$ and measured for, e.g., $\mathrm{CO}, \mathrm{O}, \mathrm{O}_{2}$, $\mathrm{NO}, \mathrm{N}$, and $\mathrm{N}_{2} \cdot{ }^{10}$

Electron structure is designed to get better catalysts both by alloying, surface structuring, growth, deposition of ultrafine particles $^{11-16}$ and by direct atom manipulation in Scanning
Tunneling Microscopy (STM) and Atomic Force Microscopy (AFM). ${ }^{17,18}$ Noble metals illustrate the delicate difference between inertness and reactivity, the noble solid being inert to atmospheric attack while used as a catalyst in reactive nanoparticle form. ${ }^{19}$

Strong and weak adsorption differ also in spatial behavior, illustrated (Fig. 1 ) by the almost reversed (left-right) potentialenergy surfaces (PES's) for Ar and CO adsorbed on a dense $\mathrm{Ni}$ surface with an adatom of Ni. The $\mathrm{CO}$ molecule adsorbs more strongly on transition metals than on noble metals, with an overall preference for low-coordination sites, as is well known from GGA calculations. ${ }^{2,8,9,20-22}$ The vdW-DF method, ${ }^{23}$ used for Fig. 1 also favors $\mathrm{CO}$ adsorption on $\mathrm{Ni}$ adatom $\left(E_{\mathrm{ads}}=\right.$ $2.2 \mathrm{eV}$ ) over terrace (interaction energies down to $1.8 \mathrm{eV}$ ). For Ar, the weak adsorption is determined by vdW forces, favoring proximity to extended surface at terrace $\left(E_{\mathrm{ads}}=0.10 \mathrm{eV}\right)$, but avoiding unnecessary Pauli repulsion, as on top of $\mathrm{Ni}$ adatom (interaction energies down to $0.06 \mathrm{eV}$ ).

We observe an atomic-scale spatial variation of the vdWforce field, which we derive with the vdW-DF method, but which we claim is a true physical phenomenon, present also in more recent DFT's with account of vdW forces. The $\mathrm{vdW}-\mathrm{DF}^{23}$ is used perturbatively from revPBE ${ }^{24}$ densities for all calculated interaction energies, unless otherwise stated. It expresses the exchange-correlation energy as $E_{x c}^{\mathrm{vdW}-\mathrm{DF}}=$ $E_{x}^{\mathrm{revPBE}}+E_{c}^{\mathrm{LDA}}+E_{c}^{\mathrm{nl}}$, i.e., with exchange accounted for by $E_{x}^{\text {revPBE}}$, local correlation by $E_{c}^{\mathrm{LDA}}$, and nonlocal correlation, and thus vdW interactions, by

$$
E_{c}^{\mathrm{nl}}=\frac{1}{2} \int d^{3} r d^{3} r^{\prime} n(\vec{r}) \phi\left(\vec{r}, \vec{r}^{\prime}\right) n\left(\vec{r}^{\prime}\right) .
$$

Equation (1) depends nonlocally on the electron density $n(\vec{r})$, the scalable interaction kernel $\phi\left(\vec{r}, \vec{r}^{\prime}\right)$ being attractive at medium-to-large separations and repulsive at small ones. ${ }^{23}$ It expresses the electron-response nature of the vdW interaction. $^{23}$

The integration in Eq. (1) is sped up by factorization and fast Fourier transforms. ${ }^{25}$

All calculations are made with the GPAW software, ${ }^{26}$ which also has a self-consistent version of the vdW-DF ${ }^{25,27}$ available. 


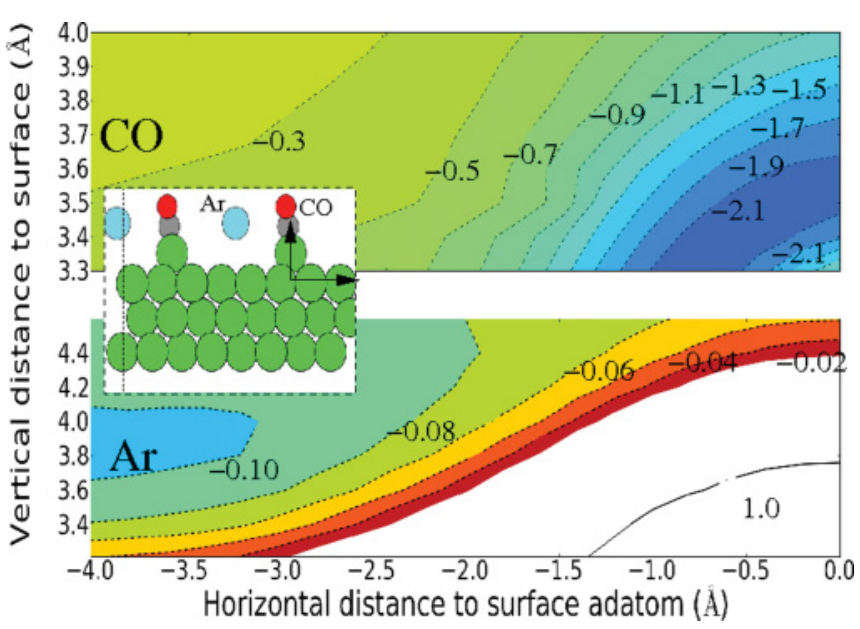

FIG. 1. (Color online) Spatial difference in interaction energy between strong and weak adsorption shown by potential-energy surfaces (PES's) for CO (top) and Ar (bottom) adsorbed on Ni(111) surface with $\mathrm{Ni}$ adatom (inset), calculated with the vdW-DF method. ${ }^{23}$ $\mathrm{CO}$ molecule prefers to be close to adatom, while the Ar atom prefers terrace, as indicated by coordinate system in inset. Units are $\mathrm{eV}$ and $\AA$.

The slab calculations are performed with orthogonal unit cells and a slab of three layers $(4 \times 3)$, when benzene is the adsorbate, and four layers $(2 \times 2)$ for CO. Default GPAW parameters are used, apart from a grid spacing of $0.18 \AA$. We use $4 \times 4 k$ points. Every point on the PEC allows benzene relaxation in plane parallel to surface. The vdW-DF parameters are as in Ref. 25. During the relaxation of slabs, the bottom layer of the slab is fixed. The height of the cell is $23 \AA$. In Fig. 1, a linear combination of atomic orbitals basis set with double-zeta-polarized precision is used for reasons of electronic spin convergence of the $\mathrm{O}$ atom. Elsewhere, the more accurate default grid mode in GPAW is used for the density.

The vdW effects are illustrated by calculated vdW-DF energy landscapes for representative molecules on a variety of substrate configurations (Fig. 2 ). PEC results for benzene adsorbed on planar $\mathrm{Ni}(111)$ surface, and $\mathrm{Ni}(111)$ surface with $\mathrm{Ni}$ adatom and pyramid, respectively, show (i) GGA (revPBE) results to follow the low-coordination rule, ${ }^{1-4}$ (ii) noticeable differences between revPBE and vdW-DF results, and (iii) significant structure-dependent differences in nonlocal correlations, $E_{c}^{\mathrm{nl}}$ (lower panel), most attractive for planar surface (several eV's), and significantly smaller for adatom and pyramid structures of the nonlocal character of the $\mathrm{vdW}$ interaction ${ }^{23}$ and easily understood from Eq. (1) and the form of the kernel $\phi\left(\vec{r}, \vec{r}^{\prime}\right)$, being attractive in key regions. ${ }^{23}$ The average separation between electrons on adsorbate and $\mathrm{Ni}$ atoms in substrate, respectively, is shortest on the planar surface, giving the strongest vdW effect, but longest for the pyramid structure, making the vdW attraction weakest here, a clear effect of the extended surface.

In weak adsorption, the outcome of the delicate competition between covalent, vdW, and Pauli-repulsion forces also depends on the nature of the substrate atoms. Benzene interaction-energy values for the three indicated structures illustrate this (Figs. 3 and 4 ). For $\mathrm{Ag}(111)$ and $\mathrm{Au}(111)$, and

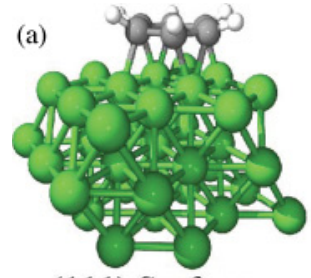

(111) Surface

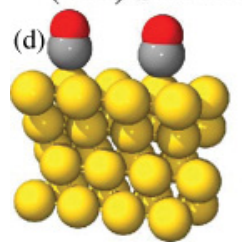

(111) Surface

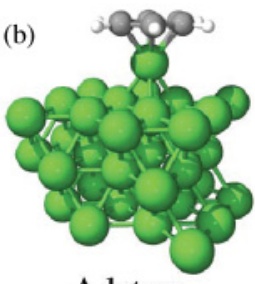

Adatom

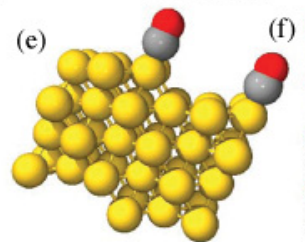

(211) Step (c)

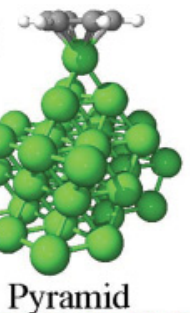

FIG. 2. (Color online) Studied substrate structures for adsorption of benzene molecule on (a) planar $\mathrm{Ni}(111)$ surface, (b) adatom, and (c) pyramid $\mathrm{Ni}$ clusters adsorbed on $\mathrm{Ni}(111)$ surface, and of $\mathrm{CO}$ molecule on (d) planar $\mathrm{Au}(111)$ and (e) stepped $\mathrm{Au}(211)$ surfaces. The adsorption sites are not equivalent, since benzene binds to several $\mathrm{Ni}$-metal atoms in planar case to only top atom on, e.g., the pyramid and adatom. The deformation of the adsorbed benzene molecule on the planar surface is indicated. The cell used for benzene (dashed square) is illustrated in (f), where circles represent the adatom defect in the lower right, and the pyramid defect in the upper right (also a common model structure for an STM tip).

marginally for $\mathrm{Cu}(111)$, vdW attraction and Pauli repulsion (lower on the surface than on the adatom and pyramid) overcome any $d$-electron-benzene hybridization, to make benzene most stable on the planar surface. This is at variance with the revPBE trend, which gives no significant binding to any structure in the $\mathrm{Au}$ and $\mathrm{Ag}$ cases, while having a

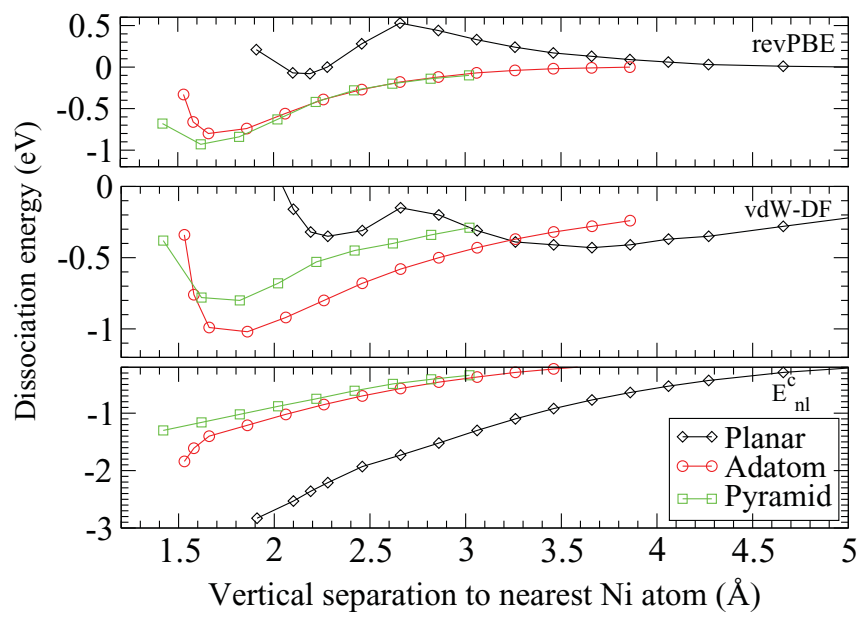

FIG. 3. (Color online) Potential-energy curves (PEC's) for benzene adsorption on planar, adatom, and pyramid structures (see Fig. 2) of $\mathrm{Ni}$, in the revPBE (top; for comparison) and the vdW-DF (middle) approximations, the coordinate $d$ being the separation between the adsorbate point of gravity and the closest metal atom (energy scales differ). The bottom figure shows a key contribution to the total vdW-DF energy, the nonlocal correlation $E_{c}^{\mathrm{nl}}$ [Eq. (1)], which is seen to be strongest for the planar surface, where the average separation between adsorbate and the $\mathrm{Ni}$ atoms is shortest, and weakest for the pyramid structure, where the average separation is longest. 


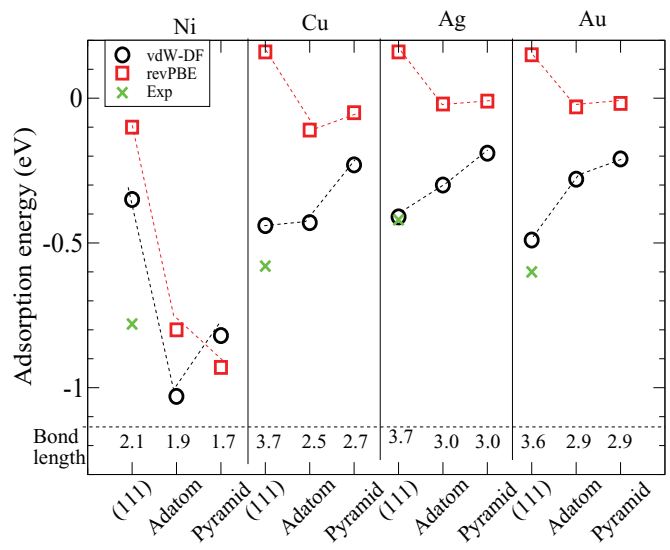

FIG. 4. (Color online) Interaction-energy values, calculated with $\mathrm{vdW}-\mathrm{DF}$ and revPBE, respectively, for benzene on $\mathrm{Ni}, \mathrm{Cu}, \mathrm{Ag}$, and $\mathrm{Au}$, for the planar (111), adatom, and pyramid configurations (described as a, b, and c in Fig. 2). Adsorption trends illustrate the competition between preference for low coordination, called for by covalency, and that for proximity to the extended surface, called for by vdW forces. Like in Fig. 3 the top panels show that the GGA revPBE (no vdW) prefers the low-coordinated adatom and pyramid sites over the planar (111) surface, for all the metals, and that the vdW-DF prefers the planar (111) surface over both adatom and pyramid, for the coinage metals. Other patterns appear, when several driving forces have comparable strengths, as illustrated by the vdW-DF case for Ni (left), where under-coordination favors the adatom over planar (111) surface and over the pyramid (due to a closer proximity to it). The force balance can be delicate, as the Ni case also illustrated in Fig. 3: The vdW-DF suggests two minima, the inner for chemisorption, the outer for physisorption. This feature is, however, functional dependent, a less repulsive exchange approximation, like the optPBE-vdW, ${ }^{47}$ slightly overestimates the adsorption and result in only one (inner) minimum at $1.1 \mathrm{eV}$ (cf. recent findings for graphene adsorption ${ }^{48}$ ). Experimental results for $\mathrm{Ni},{ }^{28} \mathrm{Cu},{ }^{29,30} \mathrm{Ag},{ }^{31,32}$ and $\mathrm{Au}^{33}$ are indicated by crosses. Bond lengths are measured from the carbon ring to the closest metal atom, in $\AA$, and listed to signal chemisorption (short) and physisorption (long).

small preference for the adatom site in the $\mathrm{Cu}$ case, and which trends on $\mathrm{Ni}$ supports the low-coordination rule, ${ }^{5,6}$ with stronger bonds on structures with lower coordination (higher $d$ band). The vdW-DF results imply that the rule does not apply for weak adsorption in presence of $\mathrm{vdW}$ forces. This can be seen from the vdW-DF altering the low-coordination trend on $\mathrm{Ni}$ predicted by revPBE, favoring the adatom structure over the pyramid. Secondly on the flat surfaces, the adsorption of benzene is slightly weaker on $\mathrm{Ag}(111)$ than on $\mathrm{Cu}(111)$ and, in particular, $\mathrm{Au}(111)$ (Fig. 4). This physisorption trend radically differs from that in strong adsorption, given by GGA for the $\mathrm{O}$ atom, whose bond is weakest on $\mathrm{Au}$ and then grows with size of the coupling matrix element $V_{a d} .^{6}$

Traditional physisorption studies ${ }^{34,35}$ involve PEC's as sums of a vdW attraction and a Pauli repulsion. The former is expressed in terms of dielectric properties of the substrate, thus relating to excitation energies of each surface. Ag has its $d$ band more than $4 \mathrm{eV}$ below the Fermi level $(2 \mathrm{eV}$ for $\mathrm{Cu}$ and $\mathrm{Au}$ ), which makes ${ }^{34,35} \mathrm{Ag}$ less polarizable and less vdW attractive (Figs. 4 and 5 ). The latter relates to the electronic

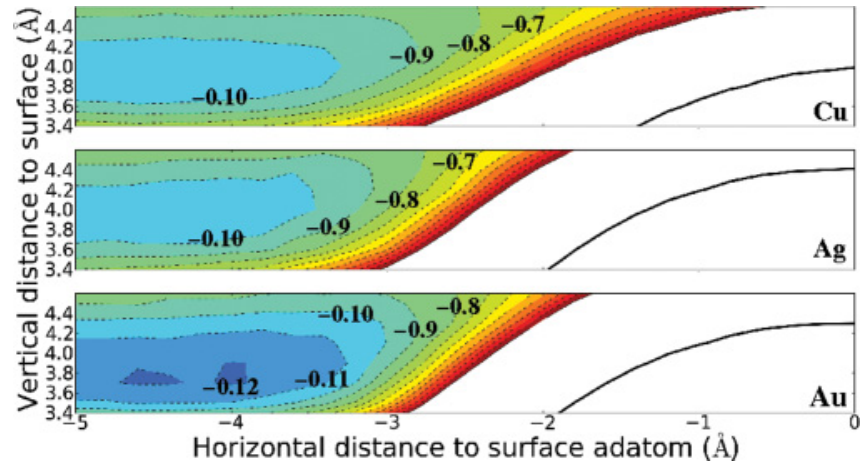

FIG. 5. (Color online) PES's for an Ar atom physisorbed on $\mathrm{Cu}, \mathrm{Ag}$, and $\mathrm{Au}(111)$ surfaces with an adatom of the same kind in the origin. The adsorption energy varies weakly between metals, Ar adsorption being slightly stronger on $\mathrm{Au}$ than on $\mathrm{Ag}$, consistent with the lower polarizability of Ag. ${ }^{34,35}$ The PES's also show the Ar preference for terrace away from the adatom. Black equipotential curve in white area, representing a $1 \mathrm{eV}$ repulsion, calculated with revPBE, illustrates the strong repulsion at small separations and the approximate correlation of these curves with the coupling matrix element $V_{a d} .{ }^{6}$ So, polarizability determines the strength of physisorptive attraction around the physisorption minima, while $V_{a d}$ gives trend for the Pauli repulsion near the substrate.

density profile at the surface and is traditionally given with an empirical input.

The variation of the vdW attraction in space is a general phenomenon $^{36}$ PES's for a single $\mathrm{Ar}$ adatom on $\mathrm{Ni}, \mathrm{Cu}, \mathrm{Ag}$, and $\mathrm{Au}(111)$ surfaces, each with a single adatom of the same kind (Figs. 1 and 5) show the typical broad range of attraction, the PES forming a shallow, semi-infinite and not so substratesensitive trough high over the surface, and steep repulsive potential, growing in strength and range (indicated by black curve in white area) in order $\mathrm{Ni}, \mathrm{Cu}, \mathrm{Ag}$, and $\mathrm{Au}$, roughly consistent with known correlation between coupling matrix element $V_{a d}$ and repulsion. ${ }^{6}$ This first-principles calculation thus both supports the qualitative picture of the traditional physisorption theories ${ }^{34,35}$ and accounts for the repulsion. For the strength and range of the latter, empirical input can be replaced by a well-founded ${ }^{6,38}$ and tabulated ${ }^{12}$ quantity, $V_{a d}$, which characterizes each atom.

For weak adsorption of CO on stepped Au surfaces, experimental data ${ }^{39-42}$ can be compared with calculated vdW-DF adsorption-energy values (Table I). Trends are right, including the preference for steps, and values are comparable. The slight underbinding can be remedied by a more favorable ${ }^{43}$ exchange functional, ${ }^{44-46}$ like the optPBE-vdW. ${ }^{47}$

TABLE I. CO adsorption-energy values (in eV) on various $\mathrm{Au}$ surface and site structures, and comparison with experiments and revPBE. Coordination number $n$ is indicated as $(n)$.

\begin{tabular}{lcccc}
\hline \hline$[\mathrm{eV}]$ & 111 & \multicolumn{2}{c}{211} & 310 \\
\cline { 3 - 4 } Site & Atop (9) & Step (7) & Terrace (9) & Step (6) \\
\hline vdW-DF & 0.10 & 0.24 & 0.14 & 0.35 \\
Exp & $<0.26^{39}$ & $0.28-0.52^{40}$ & $0.28-0.39^{41}$ & $0.48^{42}$ \\
revPBE & N.B. & 0.36 & N.B. & 0.55 \\
\hline \hline
\end{tabular}


This Brief Report exemplifies that in weak adsorption $\mathrm{vdW}$ forces can give significant changes, in particular spatial ones, by presenting results from the vdW-DF method ${ }^{23}$ for systems with carefully chosen adsorbate, substrate, and configuration. vdW effects (i) are radically more pronounced in weak adsorption than in strong, with adsorption sites and strengths that change from those for mere covalency, (ii) depend strongly on the local substrate configuration, giving strongest adsorption for proximity to extended surfaces, (iii) changes adsorption trend on, e.g., noble metals, the coupling $\mathrm{V}_{a d}$ still being relevant, but only after $\mathrm{vdW}$ and polarization properties have been considered, (iv) create a variety of behaviors, when varying kind of substrate atom and local adsorption-site structure, (v) accounts for experimental data for weak adsorption of $\mathrm{CO}$ on stepped $\mathrm{Au}$ surfaces by giving reasonably sized adsorption-energy values and right trends, including that steps are preferred, and (vi) give a first-principles support to and deeper understanding of the traditional physisorption-potential models.

The Center for Atomic-Scale Materials Design is supported by the Lundbeck Foundation.
${ }^{1}$ M. Boudart, Adv. Catal. 20, 153 (1969).

${ }^{2}$ B. Hammer, O. H. Nielsen, and J. K. Norskov, Catal. Lett. 46, 31 (1997), and references given there.

${ }^{3}$ J. T. Yates, J. Vac. Sci. Technol. A 133 (1995).

${ }^{4}$ N. P. Lebedeva, M. T. M. Koper, J. M. Feliu, and R. A. van Santen, J. Electroanal. Chem. 487, 37 (2000).

${ }^{5}$ B. Hammer and J. K. Norskov, Surf. Sci. 343, 211 (1995).

${ }^{6}$ B. Hammer and J. K. Norskov, Adv. in Catal. 45, 71 (2000).

${ }^{7}$ Z.-P. Liu, P. Hu, and A. Alavi, J Am. Chem. Soc. 124, 14770 (2002).

${ }^{8}$ T. Jiang, D. J. Mowbray, S. Dobrin, H. Falsig, B. Hvolbaek, T. Bligaard, and J. K. Norskov, J. Phys. Chem. C 113, 10548 (2009).

${ }^{9}$ L. M Molina and B. Hammer, Appl. Catal. A 291, 21 (2005).

${ }^{10}$ N. P. Lebedeva, M. T. M. Koper, J. M. Feliu, and R. A. van Santen, J. Phys. Chem. B 106, 12938 (2002), and references therein.

${ }^{11}$ M. Haruta, CATTECH 6, 102 (2002).

${ }^{12}$ A. Ruban, B. Hammer, P. Stoltze, H. Skriver, and J. Norskov, J. Mol. Catal. 115, 421 (1997).

${ }^{13}$ F. Studt, F. Abild-Pedersen, T. Bligaard, R. Z. Sorensen, C. H. Christensen, and J. K. Norskov, Science 320, 1320 (2008).

${ }^{14}$ J. Greeley, I. E. L. Stephens, A. S. Bondarenko, T. P. Johansson, H. A. Hansen, T. F. Jaramillo, J. Rossmeisl, I. Chorkendorff, and J. K. Norskov, Nature Chem. 1, 552 (2009).

${ }^{15}$ J. K. Norskov, T. Bligaard, J. Rossmeisl, and C. H. Christensen, Nature Chem. 1, 37 (2009).

${ }^{16}$ M. Haruta, Catal. Today 36, 153 (1997).

${ }^{17}$ F. E. Olsson, S. Paavilainen, M. Persson, J. Repp, and G. Meyer, Phys. Rev. Lett. 98, 176803 (2007).

${ }^{18}$ L. Gross, F. Mohn, P. Liljeroth, J. Repp, F. J. Giessibl, and G. Meyer, Science 324, 1428 (2009).

${ }^{19}$ F. Marino, C. Descorme, and D. Duprez, Appl. Catal. B: Environ. 54, 59 (2004).

${ }^{20}$ N. Lopez and J. K. Norskov, J Am. Chem. Soc. 124, 11262 (2002).

${ }^{21}$ M. Mavrikakis, P. Stoltze, and J. K. Norskov, Catal. Lett. 64, 101 (2000).

${ }^{22}$ L. M Molina and B. Hammer, Phys. Rev. B 69, 155424 (2004).

${ }^{23}$ M. Dion, H. Rydberg, E. Schroder, D. C. Langreth, and B. I. Lundqvist, Phys. Rev. Lett. 95, 109902(E) (2005).

${ }^{24}$ Y. Zhang and W Yang, Phys. Rev. Lett. 80, 890 (1998).

${ }^{25}$ G. Román-Pérez and J. M. Soler, Phys. Rev. Lett. 103, 096102 (2009).

${ }^{26}$ J. J. Mortensen, L. B. Hansen, and K. W. Jacobsen, Phys. Rev. B 71, 035109 (2005).
${ }^{27}$ T. Thonhauser, V. R. Cooper, S. Li, A. Puzder, P. Hyldgaard, and D. C. Langreth, Phys. Rev. B 76, 125112 (2007).

${ }^{28}$ A. Myers, G. Schoofs, and J. Benziger, J. Phys. Chem. 91, 2230 (1987).

${ }^{29}$ H. Koschel, G. Held, and H. Steinruck, Surf. Rev. Lett. 6, 893 (1999).

${ }^{30}$ P. Bagus, K. Hermann, and C. Woll, J. Chem. Phys. 123, 184109 (2005).

${ }^{31}$ X. Zhou, M. Castro, and J. White, Surf. Sci. 238, 215 (1990).

${ }^{32}$ R. Caputo, B. P. Prascher, V. Staemmler, P. S. Bagus, and C. Woell, J. Phys. Chem. A 111, 12778 (2007).

${ }^{33}$ D. Syomin, J. Kim, B. Koel, and G. Ellison, J. Phys. Chem. B 105, 8387 (2001).

${ }^{34}$ E. Zaremba and W. Kohn, Phys. Rev. B 15, 1769 (1977).

${ }^{35}$ P. Apell and C. Holmberg, Solid State Commun. 49, 693 (1984).

${ }^{36} \mathrm{In}$ a particular case, adsorption of $\mathrm{CO}$ on $\mathrm{Pt}(111)$, the vdWDF has been shown to destabilize the hollow site on the $\operatorname{Pt}(111)$ surface by variation of the nonlocal correlation functional [Eq. (2)] between different adsorption sites, which reduces the site-preference discrepancies in GGA. ${ }^{37}$

${ }^{37}$ P. Lazic, M. Alaei, N. Atodiresei, V. Caciuc, R. Brako, and S. Blugel, Phys. Rev. B 81, 045401 (2010).

${ }^{38}$ D. M. Newns, Phys. Rev. 178, 1123 (1969).

${ }^{39}$ M. Lazaga, D. Wickham, D. Parker, G. Kastanas, and B. Koel, ACS Symp. Ser. 523, 90 (1993).

${ }^{40}$ E. Samano, J. Kim, and B. E. Koel, Catal. Lett. 128, 263 (2009).

${ }^{41}$ J. Kim, E. Samano, and B. E. Koel, J. Phys. Chem. B 110, 17512 (2006).

${ }^{42}$ C. J. Weststrate, E. Lundgren, J. N. Andersen, E. D. L. Rienks, A. C. Gluhoi, J. W. Bakker, I. M. N. Groot, and B. E. Nieuwenhuys, Surf. Sci. 603, 2152 (2009).

${ }^{43}$ A. Puzder, M. Dion, and D. C. Langreth, J. Chem. Phys. 124, 164105 (2006)

${ }^{44}$ A. Gulans, M. J. Puska, and R. M. Nieminen, Phys. Rev. B 79, 201105 (2009).

${ }^{45}$ E. D. Murray, K. Lee, and D. C. Langreth, J. Chem. Theor. Comput. 5, 2754 (2009).

${ }^{46}$ A. K. Kelkkanen, B. I. Lundqvist, and J. K. Norskov, J. Chem. Phys. 131, 046102 (2009).

${ }^{47}$ J. Klimes, D. R. Bowler, and A. Michaelides, J. Phys. Condens. Matter 22, 022201 (2010).

${ }^{48}$ I. Hamada and M. Otani, Phys. Rev. B 82, 153412 (2010). 